\title{
A New Sparse Linear Array With Three-Level Nested Structure
}

\author{
Mingyang Chen ${ }^{1}$, Lu Gan ${ }^{2}$ and Wenwu Wang ${ }^{1}$ \\ ${ }^{1}$ Department of Electrical and Electronic Engineering, University of Surrey, U.K. \\ Emails: \{m.chen; w.wang $@$ surrey.ac.uk \\ ${ }^{2}$ Department of Electronic and Computer Engineering, Brunel University, U.K. \\ Email: Lu.Gan@brunel.ac.uk
}

\begin{abstract}
Mutual coupling, which is caused by a tight intersensor spacing in uniform linear arrays (ULAs), will, to a certain extent, affect the estimation result for source localisation. To address the problem, sparse arrays such as coprime array and nested array are considered to achieve less mutual coupling and more uniform degrees-of-freedom (DoFs) than ULAs. However, there are holes in coprime arrays leading to a decrease of uniform DoFs and in a nested array, some sensors may still be located so closely that the influence of mutual coupling between sensors remains significant. This paper proposes a new Loosely Distributed Nested Array (LoDiNA), which is designed in a three-level nested configuration and the three layers are linked end-to-end with a longer inter-element saparation. It is proved that LoDiNA can generate a higher number of uniform DoFs with greater robustness against mutual coupling interference and simpler configurations, as compared to existing nested arrays. The feasibility of the proposed LoDiNA structure is demonstrated for Direction-of-Arrival (DoA) estimation for multiple stationary sources with noise.
\end{abstract}

Index Terms-Mutual coupling, sparse arrays, nested array, uniform DoFs, LoDiNA, DoA estimation.

\section{INTRODUCTION}

Sparse array has attracted great attention in the last decade. Compared to the uniform linear arrays (ULAs), sparse arrays such as the nested array [1] and the coprime array [2] [3] have shown advantages when applied for direction-of-arrival (DoA) estimation. In applications, such as underwater acoustic detection, target tracking and environmental monitoring [4] [5], the capability of the array for DoA estimation depends on the number of the uniform degrees-of-freedom (DoFs), which is the cardinality of the maximum contiguous ULA segments in the difference coarray set, as well as the robustness against the mutual coupling, which is caused by the electro-magnetic interaction between sensors, making the signal that received by one sensor is influenced by a nearby sensor [6].

Nested array, which is formed by combining two or more ULAs with different inter-sensor spacing, can generate $O\left(L^{2}\right)$ co-array elements from $O(L)$ array elements. Compared to ULAs, the nested array is able to generate a higher number of uniform DoFs when implementing the subspace-based DoA estimation methods (MUSIC and ESPRIT [7] [8]). However, sensors in a sparse nested array may still be located so closely that the effect of mutual coupling between sensors becomes significant [9] [10]. In order to further reduce mutual coupling, a super nested array has been introduced in [11] by redistributing the elements of the dense ULA part of the nested array. In practice, the expression of such a super nested array is quite complicated. Although the dense ULA part is redistributed by increasing the distance between sensors, there still exists considerable mutual coupling and reduced DoFs of the super nested array.

Coprime array, which is presented in [2] and further improved in [12], excessively focuses on the sparsity, where the numbers of sensors in two subarrays, $M$ and $N$, are coprime to each other with different subarray inter-element spacings of $N$ units and $M$ units. This coprime array structure generates holes in its difference coarray set, inducing a smaller number of uniform DoFs than the nested arrays. In the work of [13], the coprime array with displaced subarrays (CADiS) was introduced to optimize and generalize the traditional coprime arrays. It is also extended to a linear Nested CADiS structure where $M$ and $N$ can be any positive integer numbers. This nested structure with the displacement of $N+1$ can provide the highest number of uniform DoFs. However, it suffers from the mutual coupling effect.

In this paper, we propose a new sparse linear array, named Loosely Distributed Nested Array (LoDiNA), by defining a three-level nested structure. Compared to the Super Nested Array [11], the LoDiNA structure can generate a higher uniform DoFs with less mutual coupling. The total aperture size of LoDiNA is one unit larger than that of the super nested array. LoDiNA configuration contains three linear subarrays, whereas the super nested array uses six ULAs. Compared to the Nested CADiS [13], the proposed LoDiNA can generate the same amount of uniform DoFs with less mutual coupling. The total aperture size of LoDiNA is the same as the Nested CADiS.

\section{PRELiminaries}

\section{A. Sparse Array Processing}

We consider a pair of integers $M$ and $N$. The unit interelement spacing $d$ equals $\lambda / 2$, where $\lambda$ denotes the wavelength. The array sensors are positioned at $\mathbf{p}=\left[p_{1}, \cdots, p_{L}\right]^{T}$, where $L$ is the total number of physical sensors.

Definition 1: The maximum number of the difference lags is determined by the number of unique elements in the set $\mathbb{L}_{p}=\left\{l_{p} \mid l_{p} d=p_{1}-p_{2}, p_{1} \in \mathbf{p}, p_{2} \in \mathbf{p}\right\}$. The number of elements in the difference co-array (given by the set $\mathbb{L}_{p}$ ) 

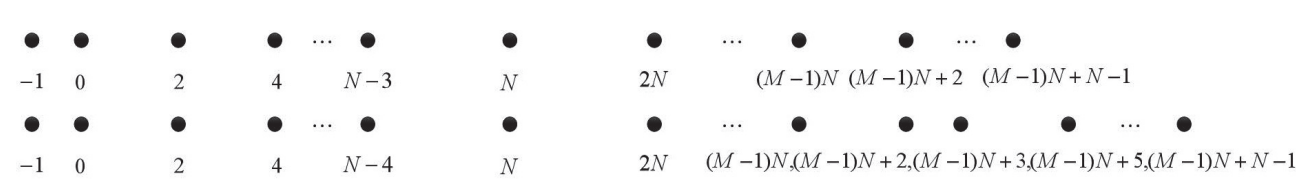

Fig. 1: The LoDiNA structure sensor locations. For the upper one, $N$ is odd, and for the lower one, $N$ is even.

directly decides the distinct values of the cross correlation terms in the covariance matrix of the signal, however, there exist pairs of $p_{1}$ and $p_{2}$ giving the same value of difference $p_{1}-p_{2}$, which causes significant mutual coupling. Thus the concept of weight function $w\left(l_{p}\right)$ is considered.

Definition 2: uniform DoFs. Let the set $\mathbb{U}$ denote the maximum contiguous ULA segments in $\mathbb{L}_{p}$. The number of elements in $\mathbb{U}$ is called the number of uniform DoFs. In this paper, in order to implement the MUSIC algorithm, we only consider the number of uniform DoFs.

Definition 3: weight function. The weight function $w\left(l_{p}\right), l_{p} \in \mathbb{L}_{p}$ of an array is defined as the number of sensor pairs which have the same value of coarray index $l_{p}$.

Assume that $D$ mono-chromatic far-field sources impinge on a sensor array, where the sensors are located at $p \lambda / 2$. Suppose that the $i$-th signal has complex amplitude $\mathbf{x}(k) \in \mathbb{C}^{D}$ at the $k$-th snapshot, where $k=1,2, \cdots, K$ and $\mathbb{C}$ represents the complex number. The DoA is denoted by $\theta_{i} \in\left[-90^{\circ}, 90^{\circ}\right]$ covering one half plane. The received sensor signal $\mathbf{y}(k) \in \mathbb{C}^{L}$ is modeled as $[\mathbf{y}(1), \mathbf{y}(2), \cdots, \mathbf{y}(K)]=\mathbf{A X}+\mathscr{N}$, where the normalized DoA of the $i$-th signal is defined as $\tilde{\theta}_{i}=$ $\left(\sin \theta_{i}\right) / 2 \in[-1 / 2,1 / 2] . \mathbf{A}=\left[\mathbf{a}\left(\tilde{\theta}_{1}\right), \mathbf{a}\left(\tilde{\theta}_{2}\right), \cdots, \mathbf{a}\left(\tilde{\theta}_{D}\right)\right] \in$ $\mathbb{C}^{L \times D}$, and the steering vector $\mathbf{a}\left(\tilde{\theta}_{i}\right)$ satisfies $\mathbf{a}\left(\tilde{\theta}_{i}\right)=$ $e^{j 2 \pi \tilde{\theta}_{i} p} \in \mathbb{C}^{L} . \mathbf{X}=[\mathbf{x}(1), \mathbf{x}(2), \cdots, \mathbf{x}(K)] \in \mathbb{C}^{D \times K}$, and $\mathscr{N}=[\mathbf{n}(1), \mathbf{n}(2), \cdots, \mathbf{n}(K)] \in \mathbb{C}^{L \times K}$, with $\mathbf{n}(k) \in \mathbb{C}^{L}$ being the independent and identically distributed (i.i.d) random noise vector. Both $\mathbf{x}$ and $\mathbf{n}$ are assumed to be vectors of zeromean, uncorrelated random variables with covariance matrices of $\mathbf{R}_{\mathbf{x x}}=E\left[\mathbf{x x}^{H}\right]$ and $\mathbf{R}_{\mathbf{n n}}=E\left[\mathbf{n n}^{H}\right]$ respectively.

The covariance matrix of data vector $\mathbf{y}$ is obtained as

$$
\mathbf{R}_{\mathbf{y y}}=\sum_{i=1}^{D} \sigma_{i}^{2} \mathbf{a}\left(\tilde{\theta}_{i}\right) \mathbf{a}^{H}\left(\tilde{\theta}_{i}\right)+\sigma^{2} \mathbf{I}
$$

where $\sigma_{i}^{2}$ is the power of the $i$-th source, $\sigma^{2}$ is the noise power and $\mathbf{I}$ is an identity matrix of dimension $\mathbb{C}^{D \times K}$. (1) can be reshaped into an autocorrelation vector $\mathbf{y}_{D}$ as

$$
\mathbf{y}_{D}=\sum_{i=1}^{D} \sigma_{i}^{2} \mathbf{a}_{\mathbb{L}_{p}}\left(\tilde{\theta}_{i}\right)+\sigma^{2} \mathbf{e}
$$

where $\mathbf{a}_{\mathbb{L}_{p}}\left(\tilde{\theta}_{i}\right) \in \mathbb{C}^{L \times K}$, e is a column vector with 1 in the middle and 0 elsewhere, and the noise $\sigma^{2} \mathbf{e}$ follows a normal distribution with zero mean. In the finite-snapshot setting, where the measurenment vectors $\mathbf{y}(k), k=1,2, \cdots, K$ are given, the covariance matrix can be estimated by

$$
\tilde{\mathbf{R}}_{\mathbf{y} \mathbf{y}}=\frac{1}{K} \sum_{k=1}^{K} \mathbf{y}(k) \mathbf{y}^{H}(k)
$$

The finite-snapshot version of the autocorrelation function can be averaged from the convariance matrix by

$$
\left\langle\mathbf{y}_{D}\right\rangle_{l_{p}}=\frac{1}{w\left(l_{p}\right)} \sum_{\left(p_{1}, p_{2}\right), p_{1}-p_{2} \in \mathbb{U}}\left\langle\tilde{\mathbf{R}}_{\mathbf{y y}}\right\rangle_{p_{1}, p_{2}}
$$

where $\left\langle\tilde{\mathbf{R}}_{\mathbf{y y}}\right\rangle_{p_{1}, p_{2}}=E\left[\langle\mathbf{y}\rangle_{p_{1}}\langle\mathbf{y}\rangle_{p_{2}}^{H}\right]$, the angle bracket $\langle\mathbf{y}\rangle_{p}$ represents the value of the signal at the sensor location $p d$, only $p_{1}-p_{2} \in \mathbb{U}$ is considered. The weight function $w\left(l_{p}\right), l_{p} \in \mathbb{U}$ has been defined earlier.

In order to estimate the DoA $\tilde{\theta}_{i}$ from $\mathbf{y}_{D}$, a variation of the rank-enhanced spatial smoothing MUSIC algorithm will be used in this paper [1] [12] [14]. The spatial smoothing step can be modified so that the finite-snapshot autocorrelation vector equals $\left\langle\mathbf{y}_{D}\right\rangle$ according to [14]. A Hermitian Toeplitz matrix $\tilde{\mathbf{R}}$ can be constructed as

$$
\langle\tilde{\mathbf{R}}\rangle_{p_{1}, p_{2}}=\left\langle\mathbf{y}_{D}\right\rangle_{p_{1}-p_{2}}
$$

where $p_{1}, p_{2} \in \mathbb{U}^{+}$, and the set $\mathbb{U}^{+}$denotes the non-negative part of the maximum contiguous ULA segment. The proof provided in [14] shows that the MUSIC spectrum over $\tilde{\mathbf{R}}$ gives the same performance as that over the spatially-smoothed (ss) matrix $\tilde{\mathbf{R}}_{s s}$, if the noise subspace is classified by the magnitudes of the eigenvalues of $\tilde{\mathbf{R}}$.

\section{B. Mutual Coupling}

In this paper, we choose the model of mutual coupling matrix $\mathbf{C}$ introduced in [11], which is approximated by a $B$ banded symmetric Toeplitz matrix in the ULA configuration.

$$
\langle\mathbf{C}\rangle_{p_{1}, p_{2}}= \begin{cases}c_{\left|p_{1}-p_{2}\right|}, & \text { if }\left|p_{1}-p_{2}\right| \leq B \\ 0, & \text { otherwise. }\end{cases}
$$

where the angle bracket $\langle\mathbf{C}\rangle_{p_{1}, p_{2}}$ represents the value of $c$ at the location $\left(p_{1}, p_{2}\right), p_{1}, p_{2} \in \mathbf{p}$ and the coupling coefficients $c_{0}, c_{1}, \cdots, c_{B}$ satisfy $1=c_{0}>\left|c_{1}\right|>\left|c_{2}\right|>\cdots>\left|c_{B}\right|$. It is assumed that the magnitudes of coupling coefficients are inversely proportional to their sensor separations [15]. The equation of the received sensor signal $\mathbf{y}(k)$ can be redesigned as $[\mathbf{y}(1), \mathbf{y}(2), \cdots, \mathbf{y}(K)]=\mathbf{C A X}+\mathscr{N}$, where $\mathbf{C} \in \mathbb{C}^{L \times L}$ is the mutual coupling matrix.

\section{LoOsely Distributed Nested ARrAys}

In this section, we present a loosely distributed nested array and its main properties considering the uniform DoFs and mutual coupling effect. The comparisons between LoDiNA and other exsiting arrays are also given. 
TABLE I: Comparison of uniform DoFs and weight function values for different array structures.

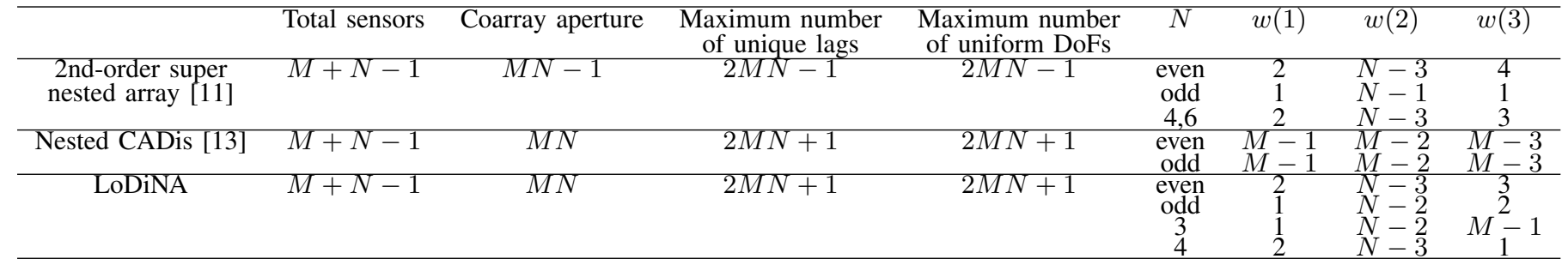

\section{A. Array Geometry}

The proposed LoDiNA is a three-level nested array, whose sensor locations are expressed as

$$
\mathbb{P}=\mathbb{S}_{1} \cup \mathbb{S}_{2} \cup \mathbb{S}_{3}
$$

where

$$
\begin{aligned}
& \mathbb{S}_{1}=\left\{-1,2 \ell_{1} \mid 0 \leqslant \ell_{1} \leqslant N_{1}\right\} \\
& N_{1}=\left\{\begin{array}{l}
\frac{N-3}{2-}, \text { odd } N \\
\frac{N-4}{2}, \text { even } N
\end{array}\right. \\
& \mathbb{S}_{2}=\left\{N \ell_{2} \mid 1 \leqslant \ell_{2} \leqslant M-1\right\} \\
& \mathbb{S}_{3}=\left\{\begin{array}{l}
N(M-1)+2 \ell_{3}, 1 \leqslant \ell_{3} \leqslant N_{21} \\
{\left[N(M-1)+2, N(M-1)+3+2 \ell_{3}\right], 0 \leqslant \ell_{3} \leqslant N_{22}}
\end{array}\right. \\
& N_{21}=\frac{N-1}{2}, \text { odd } N \\
& N_{22}=\frac{N-4}{2}, \text { even } N
\end{aligned}
$$

where $M$ and $N$ are two integers, $\mathbb{S}_{1}, \mathbb{S}_{2}$ and $\mathbb{S}_{3}$ are three levels of LoDiNA, $\ell_{1}, \ell_{2}$ and $\ell_{3}$ are three multiplication factors for different levels, with different upper limit values $N_{1}, N_{21}$ and $N_{22}$ when considering the odd and even of $N$. Figure 1 shows the sensor locations for different odd and even of $N$. From Figure 1 we can see that $\mathbb{S}_{1}$ is a nested linear array, $\mathbb{S}_{2}$ is an ULA with spacing of $N$, and $\mathbb{S}_{3}$ is another ULA with spacing of 2 when $N$ is odd, or a nested linear array when $N$ is even.

When considering the other two array structures, in order to generate the highest uniform DoFs, we choose $M=N$ for the 2nd-order super-nested array [11] and an interval of $M+1$ between $\mathbb{S}_{1}$ and $\mathbb{S}_{2}$ for the Nested CADiS structure [13].

\section{B. Properties of the LoDiNA}

To fully verify the uniform DoFs of the LoDiNA structure and the values of the weight functions, we summarize the properties of $\mathbb{L}_{p}$. The idea for proving each property is briefly introduced.

Property 1: LoDiNA has no holes in the difference coarray domain, which means its difference coarray covers a maximum consecutive virtual array positions to calculate the uniform DoFs.

Proof: The idea to prove is to assume a general linear expression for $l_{p}$ as

$$
l_{p}=N n_{0}+\ell_{0}
$$

where $0 \leq n_{0} \leq M-1$ and $0 \leq \ell_{0} \leq N-1$. It is desirable to find differences between elements in $\mathbb{S}_{1}, \mathbb{S}_{2}$ and $\mathbb{S}_{3}$ to fullfill the set of $l_{p}$ expression (8).

Property 2: The weight function $w\left(l_{p}\right)$ of the proposed LoDiNA structure with $M \geq 3, N \geq 3$ at $l_{p}=1,2,3$ is (the smaller the weight function values, the less the mutual coupling effect):

$$
\begin{gathered}
w(1)= \begin{cases}1, & \text { when } N \text { is odd, } \\
2, & \text { when } N \text { is even, }\end{cases} \\
w(2)= \begin{cases}N-2, & \text { when } N \text { is odd, } \\
N-3, & \text { when } N \text { is even, }\end{cases} \\
w(3)= \begin{cases}1, & \text { when } N \text { is } 4, \\
2, & \text { when } N \text { is odd, } \\
3, & \text { when } N \text { is even. } \\
M-1, & \text { when } N \text { is } 3 .\end{cases}
\end{gathered}
$$

Proof: The idea to prove is to list the calculation process of each weight function value directly.

\section{Comparisons with Existing Work}

Table I shows the comparison of uniform DoFs and weight function values for different array structures. When the total number of physical sensors is fixed as $L=M+N-1$, the value of uniform DoFs for the 2nd-order super nested array is 2 smaller than those of the other structures. For the Nested CADiS and LoDiNA, the numbers of uniform DoFs are the same, which is $2 M N+1$. All three sparse arrays are restricted arrays, that is, the difference coarray does not have holes, which we can see from Table I. Also in the Table I, the coarray aperture of the 2nd-order super nested array is $M N-1$, which is one unit smaller than the Nested CADiS and LoDiNA. Comparing the weight function values, we can see that when $N$ is even and $N>4$, the values of $w(1)$ and $w(2)$ are same for the 2nd-order super nested array and LoDiNA, the $w(3)$ value of LoDiNA is smaller than the 2ndorder super nested array. When $N$ is odd and $N>3$, the values of $w(1)$ are the same for the 2nd-order super nested array and LoDiNA, the $w(2)$ value of LoDiNA is smaller than the 2nd-order super nested array, and the $w(3)$ value of LoDiNA is higher than the 2nd-order super nested array. When $N$ is 4 , the $w(3)$ value of LoDiNA is smaller than the 2nd-order super nested array. When $N$ is 3, the $w(3)$ value of LoDiNA is $M-1$. Whether the value is higher than the 

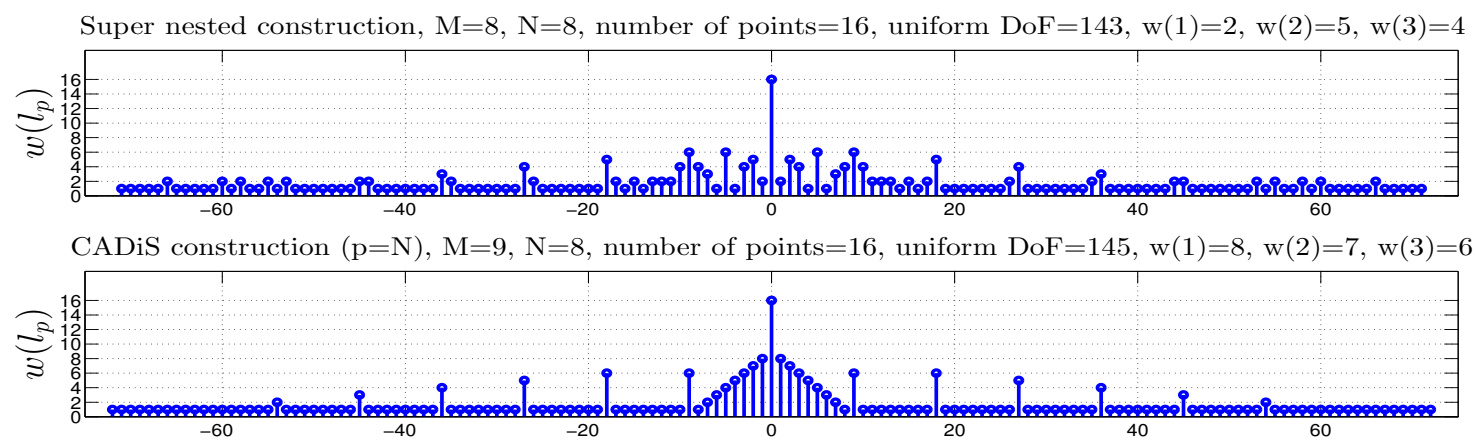

LoDiNA construction, $\mathrm{M}=9, \mathrm{~N}=8$, number of points $=16$, uniform $\operatorname{DoF}=145, \mathrm{w}(1)=2, \mathrm{w}(2)=5, \mathrm{w}(3)=3$

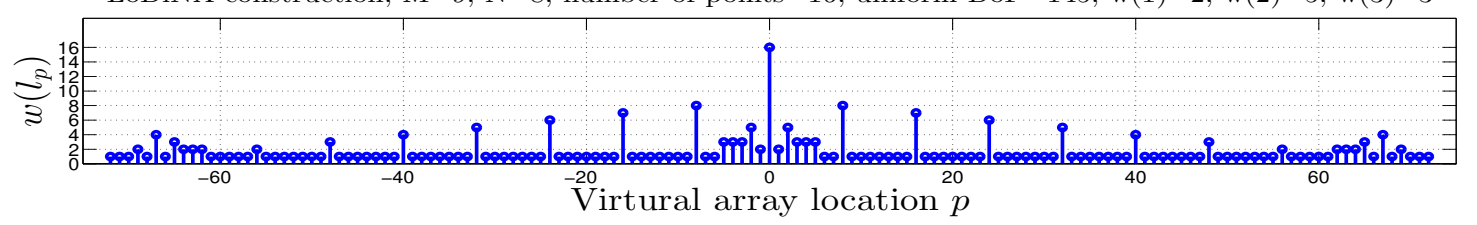

Fig. 2: The values of weight function $w\left(l_{p}\right)$ obtained by the 2nd-order super-nested arrays [11], the Nested CADiS [13], and the proposed LoDiNA structure.

TABLE II: Comparison for different array structures when using 16 sensors in the presences of RMSE of associated MUSIC spectra $P(\tilde{\theta})$ and mutual coupling, in terms of coupling leakage $(Q)$ defined as $\|\mathbf{C}-\operatorname{diag}(\mathbf{C})\|_{F} /\|\mathbf{C}\|_{F}$, where $[\operatorname{diag}(\mathbf{C})]_{i, j}=[\mathbf{C}]_{i, j} \delta_{i, j}$.

\begin{tabular}{|c|c|c|c|}
\hline & Nested CADiS & $\begin{array}{c}\text { 2nd-order } \\
\text { Super-nested }\end{array}$ & LoDiNA \\
\hline Error & 0.00146 & 0.00089 & 0.00084 \\
\hline$Q$ & 0.33477 & 0.21707 & 0.21263 \\
\hline
\end{tabular}

2nd-order super nested array depends on the choice of $M$. The weight function values of the Nested CADiS are much higher than those of the other arrays. For the configuration complexity, the super nested array has 6 levels [11], however, LoDiNA only contains 3 levels including a nested linear array, an ULA and another ULA with different inter-element spacing when $N$ is odd. When $N$ is even, the first level of LoDiNA is a nested linear array, the second level is an ULA and the third level is a nested linear array which is similar to the first level. The Nested CADiS contains 2 ULAs [13], which is 1 smaller than LoDiNA.

\section{Numerical Results}

Assume $D=30$ stationary sources whose normalized DoA are distributed at $\tilde{\theta}_{i}=-0.2+0.4(i-1) / 29, i=1,2, \ldots, 30$. The Signal to Noise Ratio (SNR) is $5 \mathrm{~dB}$ and the number of snapshots $K$ is 1000 . In order to make comparisons when using the same number of 16 physical sensors, we set $M=9$, $N=8$, and $M+N-1=16$ for the Nested CADiS [13] as well as the proposed LoDiNA structure. $M=8, N=8$, and $M+N=16$ for the 2nd-order super-nested arrays [11] to obtain a maximum number of uniform DoFs. Considering the mutual coupling defined in (6), we choose $c_{1}=0.3 e^{j \pi / 3}$ and $B=100$. The remaining coupling coefficients are given by $c_{l_{p}}=c_{1} e^{-j\left(l_{p}-1\right) \pi / 8} / l_{p}$ for $2 \leq l_{p} \leq B$. The DoAs are estimated from the measurement vectors and the MUSIC algorithm introduced in Section II is used. The associated MUSIC spectra $P(\tilde{\theta})$ and root-mean-squared error (RMSE), are computed, where the RMSE (Error) is defined as

$$
\text { Error }=\sqrt{\frac{1}{D} \sum_{i=1}^{D}\left(\hat{\tilde{\theta}}_{i}-\tilde{\theta}_{i}\right)^{2}}
$$

where $\hat{\tilde{\theta}}_{i}$ denotes the estimated normalized DoA of the $i$-th source signal, according to the root MUSIC algorithm, and $\tilde{\theta}_{i}$ is the designed normalized DoA. Note that if the mutual coupling matrix $\mathbf{C}$ is a diagonal matrix, sensor responses do not interfere with each other. Hence the coupling leakage can be defined as [11]

$$
Q=\frac{\|\mathbf{C}-\operatorname{diag}(\mathbf{C})\|_{F}}{\|\mathbf{C}\|_{F}}
$$

where $[\operatorname{diag}(\mathbf{C})]_{i, j}=[\mathbf{C}]_{i, j} \delta_{i, j}$ with $\delta_{i, j}$ being an unit matrix and $\|\cdot\|_{F}$ denotes the Frobenius norm of a matrix. The smaller the coupling leakage, the less the mutual coupling influence.

In Figure 2, we show the values of the weight functions and the maximum contiguous segments in $\mathbb{L}_{p}$. For the 2nd-order super-nested arrays in [11], the number of uniform DoFs is 143 (the sensor in coarray locates continuously from -71 to 71 ) with the weight function values $w(1)=2, w(2)=5$, and $w(3)=4$. For the Nested CADiS [13], the number of uniform DoFs is 145 (coarray locations are continuously from -72 to 72 ) with the weight function values $w(1)=8, w(2)=7$, and $w(3)=6$. For the proposed LoDiNA structure, the number of uniform DoFs is 145 (coarray locations are continuously from -72 to 72 ) with the weight function values $w(1)=2$, $w(2)=5$, and $w(3)=3$. We found that when the number of sensors are the same, the proposed LoDiNA structure can generate higher uniform DoFs with relatively smaller weight functions at $w(1), w(2)$, and $w(3)$. 
Table II shows the RMSE of the associated MUSIC spectra $P(\tilde{\theta})$ and the mutual coupling measured by coupling leakage for the Nested CADiS and 2nd-order super-nested array, and the proposed LoDiNA. We found that the proposed LoDiNA structure gives the best DoA estimate with RMSE of 0.00084 and the least mutual coupling with coupling leakage of 0.21263 . In other words, the proposed LoDiNA structure offers a higher number of uniform DoFs, and is more robust to mutual coupling effect as compared with the two baseline methods.

\section{CONClusion}

A new sparse linear array, named Loosely Distributed Nested Array (LoDiNA), has been presented. The nested structure contains three levels, which are linked end-to-end with a more loosely sensor saparation. Compared to the existing nested arrays, this new three-level nested array structure generates more uniform DoFs with greater robustness against mutual coupling effect and has a simpler configuration. The DoA estimation results show good performance of the proposed structure for multiple sources with noise.

\section{REFERENCES}

[1] P. Pal and P. Vaidyanathan, "Nested arrays: A novel approach to array processing with enhanced degrees of freedom," IEEE Transactions on Signal Processing, vol. 58, no. 8, pp. 4167-4181, 2010.

[2] P. P. Vaidyanathan and P. Pal, "Sparse sensing with co-prime samplers and arrays," IEEE Transactions on Signal Processing, vol. 59, no. 2, pp. 573-586, 2011.

[3] A. Raza, W. Liu, and Q. Shen, "Thinned coprime arrays for doa estimation," in 2017 25th European Signal Processing Conference. IEEE, 2017, pp. 395-399.

[4] J. Benesty, J. Chen, and Y. Huang, Microphone Array Signal Processing. Springer Science \& Business Media, 2008, vol. 1.

[5] M. R. Bai, J.-G. Ih, and J. Benesty, Acoustic Array Systems: Theory, Implementation, and Application. John Wiley \& Sons, 2013.

[6] M. I. Skolnik et al., "Introduction to radar systems," Mc Grow-Hill, pp. 607-609, 2001.

[7] R. Schmidt, "Multiple emitter location and signal parameter estimation," IEEE Transactions on Antennas and Propagation, vol. 34, no. 3, pp. 276-280, 1986

[8] R. Roy and T. Kailath, "ESPRIT-estimation of signal parameters via rotational invariance techniques," IEEE Transactions on Acoustics, Speech, and Signal Processing, vol. 37, no. 7, pp. 984-995, 1989.

[9] I. Gupta and A. Ksienski, "Effect of mutual coupling on the performance of adaptive arrays," IEEE Transactions on Antennas and Propagation, vol. 31, no. 5, pp. 785-791, 1983.

[10] Y. Zhang, K. Hirasawa, and K. Fujimoto, "Signal bandwidth consideration of mutual coupling effects on adaptive array performance," IEEE Transactions on Antennas and Propagation, vol. 35, no. 3, pp. 337-339, 1987.

[11] C.-L. Liu and P. Vaidyanathan, "Super nested arrays: Linear sparse arrays with reduced mutual couplingłpart i: Fundamentals," IEEE Transactions on Signal Processing, vol. 64, no. 15, pp. 3997-4012, 2016.

[12] P. Pal and P. P. Vaidyanathan, "Coprime sampling and the MUSIC algorithm," in IEEE Signal Processing Education Workshop (DSP/SPE) on Digital Signal Processing. IEEE, 2011, pp. 289-294.

[13] S. Qin, Y. D. Zhang, and M. G. Amin, "Generalized coprime array configurations for direction-of-arrival estimation," IEEE Transactions on Signal Processing, vol. 63, no. 6, pp. 1377-1390, 2015.

[14] C.-L. Liu and P. P. Vaidyanathan, "Remarks on the spatial smoothing step in coarray MUSIC," IEEE Signal Processing Letters, vol. 22, no. 9, pp. 1438-1442, 2015.

[15] B. Friedlander and A. J. Weiss, "Direction finding in the presence of mutual coupling," IEEE transactions on Antennas and Propagation, vol. 39, no. 3, pp. 273-284, 1991. 